\title{
Relativistic spacetimes and definitions of determinism
}

\author{
Juliusz Doboszewski ${ }^{1}$ (iD
}

Received: 13 April 2018 / Accepted: 22 January 2019 / Published online: 19 February 2019

(C) The Author(s) 2019

\begin{abstract}
I discuss candidates for definitions of determinism in the context of general relativistic spacetimes, and argue that a definition which does not make recourse to any particular region of spacetime should be preferred over alternatives; one such notion is discussed in detail in the light of various physical examples. The emerging picture of determinism is a pluralist one: sometimes there is no unique way of making our intuitive concept of determinism precise. Instead, what is crucial for assessment of determinism of the theory are auxiliary conditions under which it counts as deterministic or indeterministic, and justification for using them in a given situation.
\end{abstract}

Keywords General relativity $\cdot$ Spacetime $\cdot$ Determinism ·

Definitions of determinism

\section{Defining determinism}

By making spacetime dynamical, general theory of relativity challenges our everyday notions in multiple ways. Is it also posing challenges to an intuitive notion of determinism? What I take to be the intuitive notion of determinism is typically spelled out as the statement that the state of the physical system at one moment of time, together with dynamical equations describing the evolution of the system, uniquely fixes the state of the physical system at any other moment of time; this is most commonly cashed out formally in terms of a Laplacian analysis of determinism. This way of explicating determinism makes recourse to a certain spatiotemporal structure, in the sense that it makes crucial use of the notion of "moment of time" — which is a particular sort of spacetime region. What if (in some contexts) general relativity does not comply with such requirements? Indeed, in some solutions there is no sensible candidate for such a notion. Since the theory does not comply, does it even make sense to ask whether it is deterministic or indeterministic?

Juliusz Doboszewski

jdoboszewski@gmail.com

1 Institue of Philosophy, Jagiellonian University, Cracow, Poland 
The main points I will argue for are that:

1. There are multiple examples of general relativistic spacetimes suggesting indeterminism of the theory; these examples are more common, more varied, and more interesting than it is usually appreciated in the philosophy literature.

2. The dynamical nature of spacetime in general relativity implies that it is (at the very least) far from clear that available accounts of determinism are an acceptable way of explicating what determinism means. Most such accounts can well be seen as incompatible with general relativity (in the sense explicated in part Section 1.2). I catalogue possible reactions to these worries (quietism, call for a background structure, pluralism), and argue for the last one: we should accept that there are multiple equally good definitions of determinism, without privileging one over another.

3. Pluralism implies that there is no unambiguous answer to the question "is general relativity deterministic?". But that is a good thing, for it forces us to engage seriously with the task of delineating physically reasonable spacetimes from physically unreasonable ones, justification for choosing particular kind of "initial" region in a given context, and all the messy physical details associated with modeling practices.

I begin with two remarks concerning what counts as a witness of indeterminism of a theory and compatibility of an intuitive notion (or a possible formal expression of such) with a scientific theory. In Sections 2 and 3 I move on to review some of the (well-known) issues with the most popular received notion of determinism; variety of possible general relativistic examples of violations of determinism is discussed in Section 4.1. The source of trouble is identified and discussed (together with some possible reactions) in Section 4 , and an alternative interpretational story about determinism is explored in part Section 5. Section 6 summarizes.

\subsection{What is a witness of indeterminism?}

My use of the expression "witness" follows its role in mathematical logic. Recall that a witness for a formula $\varphi(x)$ with one free variable $x$ is an element $a$ such that $a$ belongs to the interpretation of the predicate $\varphi$. Similarly, a witness of indeterminism (for a given physical theory) is a subset of the set of solutions of the theory which are such that elements of this subset show that determinism is violated by the theory.

A common theme of the approaches to defining determinism I will discuss is the idea that in order to find a witness of indeterminism one has to look for pairs of models (in general relativity: spacetimes $\left\langle M, g_{a b}\right\rangle$ ) which "agree" (i.e. are isometric) in certain regions, but do not "agree" (i.e. are not isometric) overall. That is: to find indeterminism, one has to identify a region of relevant sort, and then look for multiple distinct continuations of that region. Note also that the witness is existential: in order to conclude that the given theory is indeterministic it is enough to find a single pair of models which behave in this manner; how many of such models have been found or their relative placement in the structured set of solutions is usually not considered to be important in the literature. 
A curious consequence of this way of thinking is that singular spacetimes (i.e. spacetimes containing an inextendible, incomplete causal geodesic $\gamma$ ) and indeterminism come apart. In such spacetimes it is typically not the case that $\gamma$ can be continued in more than one way; the point of singularity theorems is that there is no continuation of $\gamma$. A useful way of cashing this out is the following: the usual way of thinking about indeterminism in the philosophy literature equates indeterminism with cases where solutions do exist, but their uniqueness fails. In the case of singular spacetimes (in general) it is not the uniqueness, but the existence (for certain values of the generalized affine parameter of $\gamma$ ) of solutions which fails. So, even though singular spacetimes are intimately tied to most of the candidate examples of indeterminism I will discuss in part Section 4.1, by themselves they do not witness indeterminism. If one wishes to treat them as witnessing indeterminism, the concept of indeterminism has to be phrased differently: as the demand that some regions fix uniquely future (or past) behavior for all values of a relevant parameter, perhaps along the lines of causal determinism proposed by Hoefer (2009) (such a definition could also possibly allow for "singular" states, such as the Big Bang). But, for better or worse, this is not how matters are usually formulated in discussions concerning determinism in general relativity, and I will follow the suit.

\subsection{Two desiderata: compatibility and independence}

In what follows, I will also assume that an intuitive concept (or its explication) may be compatible or incompatible with a given physical theory. I will say that a definition $D$ is compatible with the theory if and only if some interpretation of the theoretical constructs posited by the definition can be found in all solutions of the theory. If components of the definition do not have a counterpart in some models, these models are, so to speak, invisible from the point of view of the definition; in particular, those models cannot serve as counterexamples to questions formulated with the use of $D$. In such cases the concept in question is defined merely in a subset of solutions of the theory; any solution outside that subset is irrelevant for the analysis of the concept. And it will turn out that some important notions of determinism are in this sense incompatible with general relativity.

In an ideal world one could answer the question whether a given theory is indeterministic or not without making recourse to substantial metaphysical positions. For instance, (hopefully) one does not have to choose a position on the understanding of the laws of nature in order to decide whether Einstein's field equations are wellposed for a given source term. No matter whether the best analysis of laws of nature will turn out to be the Humean, governing or powers view, EFEs will be well-posed or not. The motivation for minimizing metaphysical commitments is thus a naturalist one: the epistemic priority should be given to the scientific theory of the world, and not to a metaphysical picture of it.

Presumably, the issue of determinism cannot be totally decoupled from metaphysical questions (as witnessed by the debate concerning viability of various forms of spacetime substantivalism). However, one may nevertheless hope to avoid making substantial use of metaphysics in an analysis of determinism, and see how far this 
can be taken. This has also the advantage of opening our notion of determinism to revision in the light of various physical examples without endangering our favorite metaphysical views. Thus, I will be focusing on concepts of determinism which make minimal recourse to particular metaphysical positions; I will be using this as a heuristic in choosing notions of determinism which will be subject to analysis.

\section{The Laplacian notion...}

Intuitively, "(d)eterminism reigns when the state of the system at one time fixes the past and future evolution of the system" (Werndl 2016; see also Butterfield 2005). This intuition can be made more precise (Wüthrich 2011, following Earman 1986) in the following way:

A theory $T$ is deterministic just in case, given the state description $s\left(t_{1}\right)$ at any time $t_{1}$, the state description $s\left(t_{2}\right)$ at any other time $t_{2}$ is deducible [in principle] from $T$.

One has, then, Laplacian notion of determinism: a theory is Laplacian deterministic if and only if any two models which agree at some $t$ have to agree with each other at all $t^{\prime}$.

In the context of GR, models of the theory are pairs $\left\langle M, g_{a b}\right\rangle$, where $M$ is a smooth manifold satisfying certain topological constraints, and $g_{a b}$ is Lorentz-signature metric, coupled to matter sources by the Einstein's field equations. ${ }^{1}$ Since $g_{a b}$ is what is being solved for, it makes sense prima facie to ask whether the resulting solution has an indeterministic counterpart or not.

A version of the Laplacian notion of determinism has been prominently used in the context of the Hole argument by Earman and Norton (1987). One assumes that spacetime is maximal globally hyperbolic (i.e. there exists a Cauchy surface, that is, an achronal $n-1$-dimensional set $S$ such that every inextendible timelike curve $\gamma$ intersects $S$ exactly once, where $n$ is the dimensionality of the spacetime manifold), and considers an active diffeomorphism $d_{h}$ acting on a bounded open subset $O$ of $M$, such that $O$ is in the causal future of $S$. Transforming $\left\langle M, g_{a b}\right\rangle$ by $d_{h}$ results in a model $\left\langle M, g_{a b}^{\prime}\right\rangle$, which is isometric to $\left\langle M, g_{a b}\right\rangle$, hence observationally indistinguishable from $\left\langle M, g_{a b}\right\rangle$. However, certain metaphysical views (e.g. manifold substantivalism) are committed to the view that there is a difference between $\left\langle M, g_{a b}\right\rangle$ and $\left\langle M, g_{a b}^{\prime}\right\rangle$, concerning what happens at a particular spacetime point in $O$; more precisely, what is the value of the metric at that point. Manifold substantivalism, then, implies that GR is radically indeterministic in the Laplacian sense (see Pooley 2012 or Stachel 2014 for recent summaries of the debate and available positions). But note that this particular application of the definition only works in a limited setting of an inextendible, maximal (with respect to given initial data set) globally hyperbolic spacetime. Due

\footnotetext{
${ }^{1}$ Basic notions and notational conventions follow Manchak (2013).
} 
to these constraints, such a way of making the notion of determinism precise cannot serve in answering the general question whether GR is deterministic or not. ${ }^{2}$

\section{3 ... and its troubles}

Whether a Laplacian notion succeeds as an analysis of determinism seems to depend on the way one understands the notion of a state of the system at a moment of time. Two issues arise: first, what are these? Second, how to construct them in the way which avoids making substantial conceptual decisions pre-determining the answer to the question whether the theory is deterministic? The general worry is that if such regions do not arise in all models of the theory (but merely in some sector of the theory), it seems to make no sense to ask about determinism for these other sectors. But if so, an analysis of determinism making recourse to certain types of spacetime regions is likely to miss certain classes of models. This would be bad, since potentially important classes of solutions would be ignored. I will now show that exactly this happens in the case of the two most natural (and, to my knowledge, the only two which have been seriously considered in the context of general relativity) ways of making the notion of a "state of the system at a moment of time" precise.

One we have already seen in the context of the Hole argument: a moment of time is a Cauchy surface $S$. This demand seems to be too strong, in the sense that it by fiat rules out various interesting physical situations as irrelevant to the issue of determinism. Cauchy surfaces are useful for defining initial values and the initial value problem for the theory, where one asks about existence, uniqueness and stability of solutions to Einstein's field equations given initial data defined on a Cauchy surface. A fundamental theorem (Choquet-Bruhat and Geroch 1969) states that, given a Cauchy surface and appropriate data on it, there is a unique (up to isometry) maximal globally hyperbolic (i.e. one which cannot be enlarged without losing the property of global hyperbolicity) spacetime (i.e. development of the data) solving Einstein's field equations. So: if there is a Cauchy surface, an appropriate state on the Cauchy surface together with the laws (field equations) uniquely determines past and future. That is: the theorem implies that past and future are uniquely determined — assuming global hyperbolicity.

Some caveats are in order. First, the theorem is so far proven only for vacuum spacetimes and simplified matter models (Ringström 2009). Second, by definition, if there is a Cauchy surface in the whole spacetime (a'la the situation considered in the paragraph), then that spacetime is globally hyperbolic; so if having a Cauchy surface is necessary to ask a question about determinism, this seems to rule in favour of

\footnotetext{
${ }^{2}$ Indeed, issue of determinism of GR as conceptualized by the theoretical physics community takes the form, roughly, of asking whether there are any physically reasonable spacetimes which are not (maximal) globally hyperbolic. So assuming global hyperbolicity as a prerequisite in formulating the very question of determinism leaves one unable to address these broader issues.
} 
it. However, sometimes (for dynamical reasons or due to peculiar global features) a globally hyperbolic spacetime could be enlarged by embedding it in a non-globally hyperbolic spacetime. And that larger spacetime may be non-unique (see Thorne 1993 for an extensive discussion of a simple example, the Misner spacetime). This happens despite the fact that every spacetime has some subregion which (when considered as a spacetime on its own) has a Cauchy surface (Krasnikov 2002, proposition 17). How to reconcile this with formulation of determinism assuming Cauchy surfaces? Third, beings like us do not have epistemic access to Cauchy surfaces, and there is a precise sense in which we cannot successfully predict very much. Various general relativistic limitations of predictability have been investigated by (among others) (Geroch 1977) and Manchak (2008) and can be stated as provable no-go theorems (see Butterfield 2014 for a careful recent overview of these issues). In particular, a spatially closed globally hyperbolic spacetime may be observationally indistinguishable from one which fails to have a Cauchy surface. So being like us are limited in knowing whether they are seeing a Cauchy surface - even when they actually do so. These worries are specific to general relativity: connection between determinism and predictability in classical physics seems to be very tight (see Schmidt 1997 for the claim that classical universes are predictable, and Bishop (2003) for an overview of the vexed relationship between prediction and determinism, especially of the Laplacian variety). Thus there is a "disconnection" between determinism and predictability. Finally, it seems that one could prove similar theorems for certain non globally hyperbolic spacetimes if one assumes a boundary condition (see Reula and Sarbach 2011 for an accessible overview). So one should be vary of thinking of global hyperbolicity as necessary for determinism.

Even more worryingly, as Earman (2007) puts it, demanding that moments of time $t$ are Cauchy surfaces has all the advantages of theft over honest toil. Why is that so? Assume for a moment that $t$ in the definition has to be a Cauchy surface. This implies that the whole spacetime can be split into the product of a Cauchy surface and the real line. By the Geroch \& Choquet-Bruhat theorem, there is then a unique corresponding spacetime. Thus, this conceptual choice amounts to dismissal of most if not all potentially problematic examples, and predetermines the answer to the question whether general relativity is deterministic or not (yes, it is deterministic). Making this choice renders any subsequent considerations futile: either spacetime has a Cauchy surface (and hence its future and past are uniquely determined) or fails to have it (which means that it does not have a candidate for $t$, and cannot serve as a potential counterexample to the claim about determinism). Determinism triumphs by being smuggled into the definition as a crucial assumption. In particular, interesting physical situations which could lead to potential violations of determinism (such as (i) naked singularity formation in the gravitational collapse and (ii) extensions through Cauchy horizons) are dismissed as irrelevant on the grounds of not satisfying the premise of the definition.

Earman (2007) then proceeds to suggest that perhaps a weaker causality condition could be used. A good candidate seems to be the stable causality condition, which implies existence of a global time function, that is, a map $t: M \mapsto \mathbb{R}$ such that values of $t$ monotonically increase along all future directed timelike curves. A moment of time, then, is the spacetime region corresponding to $t=$ const hypersurface of $t$. 
Note that global time function $t$ is highly non-unique (but so are, in general, Cauchy surfaces $S$ ). Because global hyperbolicity implies stable causality (but not the other way round), this is a less restrictive condition.

But this choice also seems inappropriate, for the following reason. It turns out that there exist spacetimes which have a global time function (indeed: are maximal globally hyperbolic) in certain regions, but extensions of these regions are (i) non-unique, and (ii) do not have a global time function (because the non-unique regions have closed time-like curves; an intuitive way of thinking about some of these situations is that dynamics of the spacetime is such that from certain point onwards the time function becomes periodic). ${ }^{3}$ So a global time function does not exist for the whole spacetime. In particular, it cannot be used as a parameter in determining whether two solutions agree or disagree. This shows that the Laplacian formulation making use of stable causality is too in an unfortunate position: it does not recognize an interesting class of models of the theory as relevant to the issue of determinism.

\section{The diagnosis: mistaken commitment to a particular sort of region in spacetime}

The very formulation of Laplacian determinism makes sense only under the assumption that certain physical structure is present. It seems, then, that either

1. this makes a Laplacian notion of determinism unsuitable for general questions concerning determinism in theories with a dynamical spacetime; or

2. a believer in the Laplacian notion is forced to postulate the existence of physical structure on conceptual grounds; or

3. certain physical issues need to be settled in a particular way for the definition to be applicable (but what if it turns out that these issues will not be settled in this particular way after all?)

What should we make out of this situation? There are three main options; the first two correspond to 1 . and 2. above.

1'. One could adopt a form of quietism (which seems suggested by Earman (2007), who notes that in cases of spacetimes violating stable causality perhaps it makes no sense to ask about Laplacian determinism). Perhaps the Laplacian explication of determinism is equipped with inherent limitations (and hence, is of limited use in fundamental physics).

2'. Alternatively, commitment to particular sort of regions could be understood as an imposition of a background structure: perhaps one could argue that Laplacian

\footnotetext{
${ }^{3}$ Few different types of examples of such spacetimes are constructed by Chruściel and Isenberg (1993). Doboszewski (2017) is a recent philosophical discussion of such spacetimes. Ringström $(2009,2010)$ and Belot (2011) are some of the authors who stress that these spacetimes present us with genuine - as against gauge-like indeterminism of the sort given by the Hole argument (cf Section 2). Some of the spacetimes discussed in the context of time machines by Earman et al. (2009) are also of this type.
} 
determinism simply is a notion which requires some sort of background structure, in the form of a causality condition. ${ }^{4}$ This position would be somewhat similar to Lam (2011) interpretation of what troubles arise from the fact that there is no local, tensorial expression for gravitational energy: Lam proposes to interpret gravitational energy as a concept which requires the imposition of additional background structure (for gravitational energy, that structure is captured by boundary conditions encoding the behavior of spacetime at spatial infinity).

3'. Deny that there is one particular sort of spacetime regions required for an analysis of determinism (an option I will advocate in Section 5).

More generally, if some notion of determinism postulates the existence of certain regions in spacetime, one can practically always find spacetimes which fail to have this sort of region. Perhaps it would be better not to tie determinism to any particular sort of region in spacetime. But how to achieve that?

\subsection{Variety of ways in which GR may be indeterministic}

The view that there may be no unique choice of region for the Laplacian analysis becomes more plausible when one realizes the heterogeneity of instances of indeterminism in general relativity. For there are quite a few types of solutions which one could point to when arguing for some intuitive form of indeterminism. I feel that this is an important point, which should be particularly stressed in the light of the fact that some authors (for example Kutach 2013) seem to suggest that only naked singularities and closed time-like curves are relevant cases of violations of determinism. This is at best an inappropriate simplification. For here is a list of no less than 10 types of violations of determinism (admittedly: not all mutually exclusive), many of them discussed already in Earman (1995).

1. spacetimes which violate even very weak causality conditions (and so do not allow for anything like an initial value problem);

2. spacetimes with closed time-like curves, especially spacetimes with time machines, in which chronology-respecting region is followed by a chronologyviolating region (some of the spacetimes discussed in the second caveat of part Section 3 belong to this category; another example is the Goedel solution);

3. Malament-Hogarth-type spacetimes with space invaders, in which (say) radiation can arrive to the system from infinity (Earman and Norton 1993 is a classic discussion);

4. spacetime with Cauchy surface can be "not as large as possible", and can be extended: such extensions may or may not be unique, and may or may not satisfy some causality condition (again, spacetimes discussed in part Section 3 are an example of this; but the maximally extended Kerr spacetime also is);

\footnotetext{
${ }^{4}$ Causality conditions may be thought of as constraints on the behavior of lightcone structure of spacetime (for instance, whether a spacetime allows for closed causal loops or split into time and space). See Minguzzi and Sánchez (2008) for an authoritative introduction to the importance of the causal hierarchy.
} 
5. spacetimes with various types of holes (Manchak 2014);

6. spacetimes in which spacetimes manifold exhibits exotic smooth structure (Etesi 2015);

7. white holes, i.e. time reversed counterparts of black holes (Penrose 1979);

8. naked singularities may form dynamically in gravitational collapse;

9. Cauchy horizons may form dynamically in cosmological contexts and black hole solutions;

10. finally, there is are few ways in which one could argue that globally hyperbolic spacetimes are rare and atypical in the set of all relativistic spacetimes.

On one hand, all these spacetimes seem (intuitively) relevant to the issue of determinism: On the other hand, these spacetimes have very little causal structure in common. Some of them have closed time-like curves, others do not. In some, achronal subsets have non-empty domains of dependence, in some an achronal subset cannot be chosen. Some admit a global time function, some admit it in certain regions only, and some not at all. And so on. And one could very well be worried that dismissing some of these from the subset of spacetimes relevant to determinism may amount to stacking the chances in favor of determinism.

Where is this plethora of examples coming from? Partly it comes from the dynamical nature of spacetime: changes in the source terms lead to changes in geometry. But our tenuous grip of what characterizes a physically reasonable spacetime could also be blamed, in the following sense.

When one faces a candidate witnesses of indeterminism, one should ask whether the witness is genuine, i.e. is a physically significant example. The answer to this question partly depends on what exactly is one's notion of determinism, and partly on one's assessment of that particular solution. For the first one, the issue of specifying "moments of time" (or region $\mathbf{S}$ in the definition I consider in Section 5) is crucial; for if solutions in a candidate set do not have a suitable representation of "moments of time", the set cannot be a genuine witness of indeterminism.

The subsequent question is: if one has a set of witnesses of indeterminism, which of these truly represent physically possible worlds (according to GR), and which are devoid of physical significance, being just mathematical constructions of pairs $\langle M, g\rangle$ ? This is a form of question what is a "physically reasonable" spacetime (cf Manchak 2011). And the choice of spacetime properties delineating reasonable models from unreasonable ones is a delicate manner: for it very easily tips the balance in favor of either determinism (causal condition leading to the "theft" of part Section 3) or indeterminism (admissibility of holes in the "dirty open secret" of section 5.3 of Earman 1995).

Such considerations do not seem to be captured by the received Laplacian analysis of determinism, which I believe decreases its heuristic value. But they can be captured by a closely related notion I will discuss in the next section.

\section{The remedy: pick the most flexible definition}

Consider now the following definition due to Butterfield (1989): 
(A) theory with models $\left\langle M, O_{i}\right\rangle$ is $\mathbf{S}$-deterministic, where $\mathbf{S}$ is a kind of region that occurs in manifolds of the kind occurring in the models, iff: given any two models $\left\langle M, O_{i}\right\rangle$ and $\left\langle M^{\prime}, O_{i}^{\prime}\right\rangle$ containing regions $S, S^{\prime}$ of kind $\mathbf{S}$ respectively, and any diffeomorphism $\alpha$ from $S$ onto $S^{\prime}$ : if $\alpha^{*}\left(O_{i}\right)=O_{i}^{\prime}$ on $\alpha(S)=S^{\prime}$, then: there is an isomorphism $\beta$ from $M$ onto $M^{\prime}$ that sends $S$ to $S^{\prime}$, i.e. $\beta^{*}\left(O_{i}\right)=O_{i}^{\prime}$ throughout $M$ and $\beta(S)=S^{\prime}$.

This definition appears in the context of the debate concerning the Hole argument, and shares many features with the Laplacian notion of determinism. In particular, the witness of determinism is a pair of models of the theory which agree in certain regions and disagree in certain other regions. But it makes no explicit recourse to something like a state of the physical system defined at a moment of time. Granted: this is one possible way of spelling out what the $\mathbf{S}$-regions are, and I am not saying that in certain contexts this is not a right choice to make; perhaps some features of the situation one is interested in justify that particular choice. But the definition does not force us to spell out what $\mathbf{S}$-regions are in this particular way.

This definition has four pleasant features, which I will denote by Gauge, Flexibility, Generality, and Actual Use.

Gauge: The definition is designed so that it allows for gauge freedom, by explicitly including transformations $\alpha$ (which, in the case of general relativity, will be a subset of the set of all diffeomorphisms of the spacetime manifold $M$ ).

Flexibility: The definition contains unspecified notions, and is akin to a definition scheme, in that it does not make any precise demand on what the $\mathbf{S}$ regions are. This allows one to observe that even though some choices of $\mathbf{S}$ will trivialize the issue of determinism, some other choices will not; and consequently, one is allowed to say things like: "general relativity is Cauchy surface deterministic (because of Choquet-Bruhat \& Geroch theorem) and it is maximal globally hyperbolic development indeterministic (because there are spacetimes with nonunique, non-globally hyperbolic extensions of the maximal globally hyperbolic development)".

Generality: The definition is easy to generalize. That is, one can easily modify it by requiring that the models $\left\langle M, O_{i}\right\rangle$ satisfy some auxiliary assumptions, and make variation of these assumptions explicit; for instance, one could require that

(a) some or all of the models satisfy certain additional conditions, such as energy conditions, symmetries, value or sign of cosmological constant, global properties (e.g. hole freeness condition, asymptotic behavior, causality conditions or $p$-inextendibility ${ }^{5}$ ), and so on;

(b) functions $\alpha, \beta$ satisfy additional constraints, for instance preservation of some spacetime structure (like temporal or spatial orientation), being a transformation which is in some sense close to the identity, or leaves some Cauchy surface $S$ unchanged (see Chruściel and Isenberg (1993) for an instructive use of such conditions). In this way one can generate more fine-grained equivalence relations on the set of models one is interested in;

\footnotetext{
${ }^{5}$ See Manchak (2016b) for a discussion of $p$-inextendibility.
} 
(c) S-regions are stable against some type of perturbations (in a sense to be made precise) ${ }^{6}$

Actual Use: A similar notion (under one particular way of filling in the blanks, i.e. with $\mathbf{S}$-region being the maximal globally hyperbolic development) seems to be actually used by physicists. Let me elaborate on this point. In the context of the search for solutions to the initial value problem one asks whether the maximal globally hyperbolic development of given initial data is extendible or not. Indeterminism is identified with existence of extensions. Since existence of such extensions can be demonstrated, the moral is Ringström (2010) “that Einstein's general theory of relativity is not deterministic; given initial data, there is not necessarily a unique corresponding universe". This conclusion cannot be reached using a Laplacian notion with either Cauchy surface or a value of the cosmic time function playing the role of the moment of time (for reasons discussed in part Section 3). But it is justified if $\mathbf{S}$ is the whole maximal globally hyperbolic development. Note that such states are not intrinsic in the sense of Butterfield (2005), for they contain some information about the temporal precedence relation between distinct elements of the state. This information, however, may be only partial (information concerning events in the non-globally hyperbolic part is not encoded in the information concerning the globally hyperbolic part). So it seems that in general relativity non-intrinsic states, such as maximal spatiotemporal regions satisfying certain conditions (such as being determined by the initial data) not only are a reasonable choice of the $\mathbf{S}$-region, but are a choice which is actually made - or, at least, a choice which provides an plausible interpretation of reasoning which underlying conclusions concerning failure of determinism in the context of the initial value problem made by certain physicists.

Making use of Generality, a modified definition scheme can be written down:

a theory with models $\left\langle M, O_{i}\right\rangle$ is $\mathbf{S}$-deterministic, where $\mathbf{S}$ is a kind of region that occurs in manifolds of the kind occurring in the models, iff given any two models $\left\langle M, O_{i}\right\rangle$ and $\left\langle M^{\prime}, O_{i}^{\prime}\right\rangle$ containing regions $S, S^{\prime}$ of kind $\mathbf{S}$ respectively, such that $\left\langle M, O_{i}\right\rangle$ satisfies set of conditions $C$ and $\left\langle M^{\prime}, O_{i}^{\prime}\right\rangle$ satisfies set of conditions $C^{\prime}$, and any diffeomorphism $\alpha$ from $S$ onto $S^{\prime}$ such that $\alpha$ satisfies set of conditions $C_{\alpha}$, if $\alpha^{*}\left(O_{i}\right)=O_{i}^{\prime}$ on $\alpha(S)=S^{\prime}$, then there is an isomorphism $\beta$ from $M$ onto $M^{\prime}$ that satisfies set of conditions $C_{\beta}$ which sends $S$ to $S^{\prime}$, i.e. $\beta^{*}\left(O_{i}\right)=O_{i}^{\prime}$ throughout $M$ and $\beta(S)=S^{\prime}$.

\footnotetext{
${ }^{6}$ This, potentially, allows to make a connection between some versions of the strong cosmic censorship and determinism. Namely, strong cosmic censorship is sometimes (Ringström 2010) cashed out in the following way: initial data sets with the property that their maximal globally hyperbolic developments allow non-unique extensions are rare in the set of all solutions. Recall that under a standard notion of determinism the witness is existential, which implies that stability is irrelevant for determinism - all that matters if finding a pair of models which violate determinism. That verdict will be unchanged by rarity of such pairs in the set of all models. So strong cosmic censorship and the issue of determinism decouple under the standard notion, but do not have to under the proposal I am advocating for.
} 
An advantage of that scheme is the explicit use of the sets of conditions $C, C^{\prime}, C_{\alpha}$ and $C_{\beta} \cdot{ }^{7}$

Accepting this definition scheme leads to a form of pluralism about definitions of determinism. There is no single concept of determinism; rather, there is a plethora of equally valid ways of making the notion of determinism precise. Under some of these GR will be deterministic; under some other, GR will be indeterministic. The interesting question is not so much whether GR is deterministic or not, but rather under which conditions some form of indeterminism arises, and which sets of conditions are useful in modeling a given class of physical phenomena. For instance, sometimes one may pick as region $\mathbf{S}$ a Cauchy surface; in other contexts, one may ask about the maximal globally hyperbolic development; in yet another case, initial data could be defined on a null hypersurface. All these choices are equally valid (albeit some may seem more natural to us). Which ones are more relevant to the issue of determinism depends (in large part) on which auxiliary conditions have physical significance and on the pragmatics of the application of the theory.

\section{Summary}

Let me repeat and sharpen some of the points I have made above, by presenting them in a form of three advices concerning how to think about determinism in general relativity and other physical theories.

First: in a highly mathematized theory (such as general relativity) plenty of ways of making our intuitions concerning determinism precise are available. There may very well be no single, unified concept of determinism - the most useful "definition" could merely be a convenient scheme. Rather than insisting on this or that particular definition, it seems more fruitful to ask under which auxiliary assumptions one could find a witness of indeterminism in this or that precise sense, and investigate mathematical, physical and metaphysical significance of these auxiliary assumptions.

Second: instead of saying "GR is deterministic, period", or "GR is indeterministic, case closed", one is better off saying things like: GR is Cauchy surface and Cauchy-inextendible deterministic ${ }^{8}$, maximal global hyperbolic development and inextendible indeterministic ${ }^{9}$, maximal global hyperbolic development and epistemic hole freeness deterministic ${ }^{10}$, and so on. Thus, there is no unambiguous way of determining which examples of the Section 4.1 are genuine witnesses of indeterminism.

\footnotetext{
${ }^{7}$ In most cases one will likely set $C=C^{\prime}$ and $C_{\alpha}=C_{\beta}$, but one could possibly privilege a given model by demanding less from it than from all the other alternatives. For instance, one may ask whether there exist non-isometric stably causal conunterparts to a given globally hyperbolic spacetime.

${ }^{8}$ Because if the $\mathbf{S}$ region is chosen to be a Cauchy surface and the auxiliary conditions $C=C^{\prime}$ are Cauchyinextendibility, then the spacetime is unique by the Geroch and Choquet-Bruhat theorem discussed in Section 3.

${ }^{9}$ Because the maximal globally hyperbolic development given uniquely by the Geroch and ChoquetBruhat theorem can be extendible, and such extensions can fail to be unique.

${ }^{10}$ Insofar as all known cases of non-unique extensions of previous footnotes have epistemic holes (in the sense of Manchak 2016a).
} 
But auxiliary conditions can capture the sense in which given class of spacetimes is or is not a physically reasonable one, and hence more or less important in given physical context. ${ }^{11}$ To paraphrase (Malament 2008), the interesting question is whether and in what sense examples I presented in part Section 4.1 are examples of physically reasonable general relativistic systems. In this sense pluralist take on determinism in general relativity is not a form of quietism.

Third: notions of determinism which insist on a unified, restrictive concept are likely to miss or misrepresent interesting physical situations (in particular, developing a formal notion intended to capture some forms of determinism is likely to tie one to a commitment to some particular sort of spacetime regions). One should rather be flexible: attention to physics may force us to revise our pre-theoretical notions in the light of novel physical possibilities presented to us by our best theories.

Acknowledgments The author is financially supported by a doctoral scholarship of the Polish National Science Centre, grant number 2017/24/T/HS1/00315. I am grateful to Jeremy Butterfield, Erik Curiel, Jerzy Gołosz, Aleksandra Samonek, Christian Wüthrich, and two anonymous referees of this journal as well as audiences in Bristol, Geneva and Salzburg for discussion and/or comments on previous versions of this article.

Open Access This article is distributed under the terms of the Creative Commons Attribution 4.0 International License (http://creativecommons.org/licenses/by/4.0/), which permits unrestricted use, distribution, and reproduction in any medium, provided you give appropriate credit to the original author(s) and the source, provide a link to the Creative Commons license, and indicate if changes were made.

Publisher's note Springer Nature remains neutral with regard to jurisdictional claims in published maps and institutional affiliations.

\section{References}

Belot, G. (2011). Background-Independence. Gen. Rel. Grav, 43, 2865-2884.

Bishop, R.C. (2003). On separating predictability and determinism. Erkenntnis, 58(2), 169-188.

Butterfield, J. (1989). The hole truth. British Journal for the Philosophy of Science, 40(1), 1-28.

Butterfield, J. (2005). Determinism And indeterminism. Routledge Encyclopedia of Philosophy. London: Routledge. Retrieved March, 7:2008.

Butterfield, J. (2014). On under-determination in cosmology. Studies In History and Philosophy of Science Part B: Studies In History and Philosophy of Modern Physics, 46, 57-69.

Choquet-Bruhat, Y., \& Geroch, R. (1969). Global aspects of the Cauchy problem in general relativity. Communications in Mathematical Physics, 14(4), 329-335.

Chruściel, P.T., \& Isenberg, J. (1993). Nonisometric vacuum extensions of vacuum maximal globally hyperbolic spacetimes. Physical Review D, 48(4), 1616.

Doboszewski, J. (2017). Non-uniquely extendible maximal globally hyperbolic spacetimes in classical general relativity: A philosophical survey. In Making it Formally Explicit: Probability, Causality and Indeterminism (pp. 193-212): Springer.

Earman, J. (1986). A primer on determinism. Dordrecht: D. Reidel.

\footnotetext{
${ }^{11}$ It would be very convenient if such questions could be expressed using simple formal conditions. But it could also turn out that being physically reasonable spacetime is a highly pragmatic notion, which does not anything to do with a formal condition. Hopefully, applicability of the definition scheme I advocate for does not seem to depend on a particular way in which the notion of physically reasonable spacetime is being cashed out.
} 
Earman, J. (1995). Bangs, crunches, whimpers, and shrieks: singularities and acausalities in relativistic spacetimes. New York: Oxford University Press.

Earman, J. (2007). Aspects of determinism in modern physics. In Philosophy of Physics (pp. 1369-1434): Elsevier.

Earman, J., \& Norton, J. (1987). What price spacetime substantivalism? the hole story. British Journal for the Philosophy of Science, 38(4), 515-525.

Earman, J., \& Norton, J.D. (1993). Forever is a day: Supertasks in Pitowsky and Malament-Hogarth spacetimes. Philosophy of Science, 60(1), 22-42.

Earman, J., Smeenk, C., Wüthrich, C. (2009). Do the laws of physics forbid the operation of time machines? Synthese, 169(1), 91-124.

Etesi, G. (2015). Exotica or the failure of the strong cosmic censorship in four dimensions. International Journal of Geometric Methods in Modern Physics, 12(10), 1550121.

Geroch, R. (1977). Prediction in general relativity. In Foundation of Space-Time Theories (pp. 81-93).

Hoefer, C. (2009). Causal determinism. Stanford Encyclopedia of Philosophy.

Krasnikov, S. (2002). No time machines in classical general relativity. Classical and Quantum Gravity, $19(15), 4109$.

Kutach, D. (2013). Causation and its basis in fundamental physics. London: Oxford University Press.

Lam, V. (2011). Gravitational and nongravitational energy: The need for background structures. Philosophy of Science, 78(5), 1012-1023.

Malament, D.B. (2008). Norton's slippery slope. Philosophy of Science, 75(5), 799-816.

Manchak, J.B. (2008). Is prediction possible in general relativity? Foundations of physics, 38(4), 317-321.

Manchak, J.B. (2011). What is a physically reasonable space-time? Philosophy of Science, 78(3), 410-420.

Manchak, J.B. (2013). Global spacetime structure. In Batterman, R. (Ed.) The Oxford handbook of philosophy of physics (pp. 587-606): Oxford University Press.

Manchak, J.B. (2014). On space-time singularities, holes, and extensions. Philosophy of Science, 81(5), 1066-1076.

Manchak, J.B. (2016a). Epistemic "holes" in space-time. Philosophy of Science, 83(2), 265-276.

Manchak, J.B. (2016b). Is the universe as large as it can be? Erkenntnis, 81(6), 1341-1344.

Minguzzi, E., \& Sánchez, M. (2008). The causal hierarchy of spacetimes. In Recent developments in pseudo-Riemannian geometry. ESI Lect. Math. Phys., p. 299-358.

Penrose, R. (1979). Singularities and time-asymmetry. In General Relativity: An Einstein centenary survey (pp. 581-638).

Pooley, O. (2012). Substantivalist and relationalist approaches to spacetime. In Batterman, R. (Ed.) The Oxford Handbook of Philosophy of Physics.

Reula, O., \& Sarbach, O. (2011). The initial-boundary value problem in general relativity. International Journal of Modern Physics D, 20(05), 767-783.

Ringström, H. (2009). The Cauchy problem in general relativity. European Mathematical Society.

Ringström, H. (2010). Cosmic censorship for Gowdy spacetimes. Living Reviews in Relativity, 13(2). https://doi.org/10.12942/lrr-2010-2.

Schmidt, J.H. (1997). Classical universes are perfectly predictable! Studies in History and Philosophy of Science Part B: Studies in History and Philosophy of Modern Physics, 28(4), 433-460.

Stachel, J. (2014). The hole argument and some physical and philosophical implications. Living Rev. Relativity, 17(1), 1.

Thorne, K. (1993). Misner space as a prototype for almost any pathology. In Hu, B.L., Ryan, M.P., Vishveshwara, C.V. (Eds.) Directions in General Relativity: Papers in Honor of Charles Misner, (Vol. 1 pp. 333-346): Cambridge University Press.

Werndl, C. (2016). Determinism and indeterminism. In Humphreys, P. (Ed.) The Oxford Handbook of Philosophy of Science: Oxford University Press.

Wüthrich, C. (2011). Can the world be shown to be indeterministic after all? In Probabilities in Physics, edited by Claus Beisbart, and Stephan Hartmann. Oxford: Oxford University Press. 